\title{
Custom integer optimization method for wire bundle dimensioning
}

\author{
A. Rius-Rueda*†, A. Garcia* and M. A. Díaz \\ *Universitat Politècnica de Catalunya (UPC), Barcelona, Spain \\ garciae@ee.upc.edu \\ ${ }^{\dagger}$ RückerLypsa SL, Barcelona, Spain \\ extern.armand.rius@seat.es \\ ¥Seat S.A., Barcelona, Spain \\ manuel-alberto.diaz@seat.es
}

\begin{abstract}
Automotive wiring harnesses have gained weight and complexity through the last decades due to the increasing number of electrical components, which has raised the interest on its weight optimization. For this purpose, it is essential to know at least the maximum amount of steady current that either single wires and bundles can carry. However, the large amount of combinations of the customer-specific wire harnesses makes it impossible to exhaustively simulate all of the combinations that would allow for a reliable analysis and optimization of the network. The proposed approach consists of achieving accurate predictions of the wire thermal behaviour using fast on-line polynomial functions, which have been created as regression models using data from off-line worst-case finite element simulations. These regression models provide good accuracy for the critical dimensions of wire bundles in a much faster time than simulations, so that they can be used on-line in optimization algorithms. Two different approaches of optimization are presented here in order to assign discrete values of available wire cross-section to the wire bundles: The first one uses integer linear programming, and the second one consists of a recently created custom algorithm whose objective is to reduce the computation time of the integer linear programming approach. This latter objective is satisfactorily accomplished. Results of both of the optimization approaches are validated by means of final finite element simulations, and they promisingly fulfill the objectives of this study.
\end{abstract}

Index Terms-Modular wiring, optimization, wire bundles, regression models

\section{INTRODUCTION}

The fact that some European automotive brands offer flexible possibilities of making custom configurations for their passenger cars, along with their large and increasing quantity of electrical components, has made complexity of wiring harnesses a main obstacle when it comes to optimization approaches. The overall number of possible combinations of features of a car model has been estimated to reach a magnitude around $10^{10}$.

There are simulators capable of predicting both the steady and transient temperature of wires and wire bundles ( [1], [2], [3], [4]), with the aim of either assessing the correct dimensions of wires or using them to find their optimum crosssections. It is possible to simulate all of the wires of a car if they are considered one by one, but, on the other hand, if one wants to study the temperature as a result of simultaneous current conduction in wire bundles, it is necessary to evaluate different combinations of modules in order to find the worst case. The enormous number of combinations makes it complex and, in general, not completely useful. Nonetheless, one can approach to this objective if a faster method is found.

\section{SCOPE}

Since wiring harnesses are generally assembled manually, it is impossible to assume any internal distribution of wires in bundles. Instead, one must always observe the worst case. For this purpose, all simulated bundles and the derived formulae and optimization algorithms will assume constant current density in bundles. If this is accomplished, given a set of wires, their position inside the bundles can only produce small variations on the maximum temperature. This assumption demands that all bundles have homogeneity in their heat generation, viewed as the $f$ parameter $\left(\mathrm{W} / \mathrm{m}^{3}\right)$ in the heat dissipation equation, which must be roughly uniform all over the cross-section of any random wire bundle. $f$ equals to zero in all regions of a wire bundle except in conductor regions, whose Joule effect heat depends on their current density $J$ and resistivity, $\rho$, and responds to the following equation:

$$
f=J^{2} \rho(T)
$$

The latter equation presents the resistivity of copper as a function of the temperature, $T$. Since the resistivity $\rho$ is related to the conductor material, if we want to keep $f$ uniform over a cross-section of a bundle containing wires with different conductor materials, $J$ will have to be adapted to each of them so that $f$ is approximately uniform. The different dependency of $\rho$ on the temperature for different materials complicates this, but it is still possible to find all the values of $J$ assuming a given temperature, which could be a certain point of temperature between the ambient temperature and the maximum expected temperature of the bundle. This document only explores the case of bundles with unique composition of materials (copper and polyvinylchloride). Nonetheless, power wire bundles will frequently come along with signal wires carrying negligible currents, which prevent us to maintain 


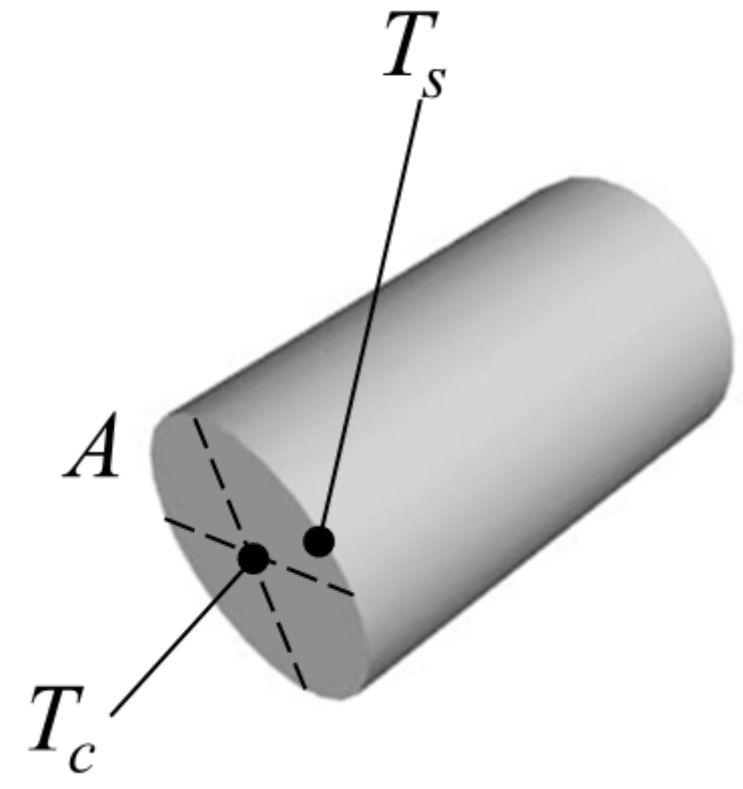

Figure 1. Ideal cylindrical conductor exposed to air, with uniform current density $J$

this desired unique Joule heat per unit volume, $f$, for all of the wires. These signal wires will have nearly zero heat dissipation in their cores, since the wire manufacturers cannot provide wires with unlimitedly thin diameters. No matter how low the current might be, these wires will always be cold, i.e. providing nearly $f=0$ heat to the system. They lack any contribution of heat to the system and their presence makes the diameter of the bundle increase, slightly allowing more heat dissipation by means of convection and radiation. These aspects make us understand that these cold wires can only benefit bundles by lowering their maximum expectable temperature for given currents. Since wire dimensioning must be done according to a reasonable worst case, this document deals only with bundles containing merely power wires with approximately uniform current density, $J$.

\section{CRITICAL DimEnSiONS OF A HOMOGENEOUS CONDUCTIVE CYLINDER WITH UNIFORM CURRENT DENSITY}

If all of the wires in the bundle are made of the same materials and they have the same current density, the temperature of the centre will increase with the radius of the bundle. It is discussed in [5] how to obtain expressions of the particular case of a cylindrical conductor exposed to air (figure 1), which is compared to wire bundles in order to validate the obtained curves of the regression models. The resulting equations 2 and 3 are plotted in figures 2 and 3.

$$
T_{\text {centre }}=f\left(\frac{A}{4 \pi \lambda}+\frac{\sqrt{A}}{2 \tilde{\alpha} \sqrt{\pi}}\right)+T_{a m b}
$$

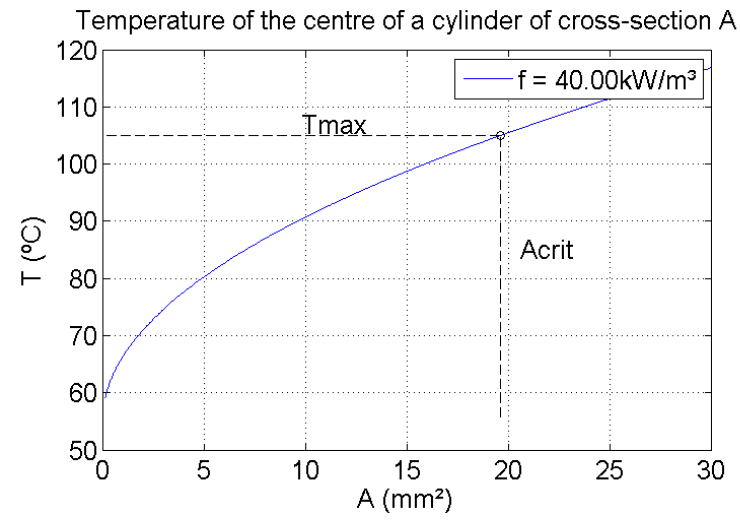

Figure 2. Centre temperature of a conductive cylinder with uniform current density and cross-section $A$

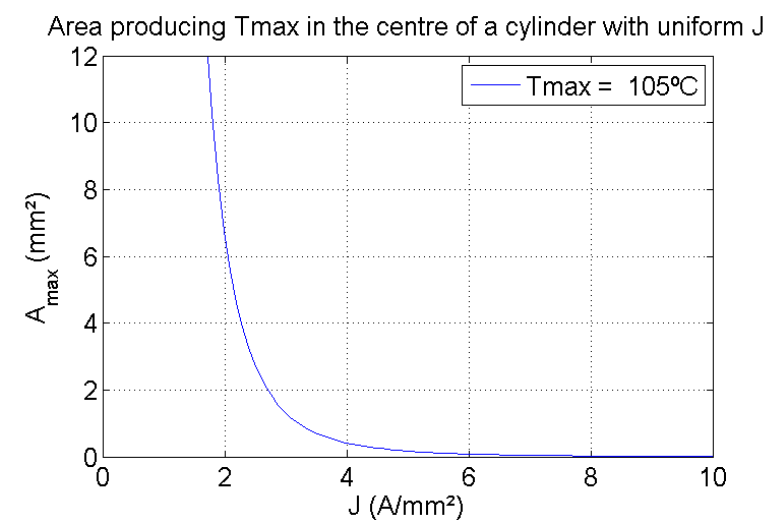

Figure 3. Maximum area of a cylindrical conductor set to current density $J$

$$
A_{\max }=4 \pi \lambda^{2}\left(\frac{-\lambda}{2 \tilde{\alpha}}+\sqrt{\frac{1}{4 \tilde{\alpha}^{2}}+\frac{T_{0}-T_{a m b}}{\lambda J^{2} \rho}}\right)^{2}
$$

Where $\lambda$ is the thermal conductivity of the cylinder material, and $r$ and $L$ are respectively its radius and its length. $\tilde{\alpha}$ is the averaged convection and radiation term of heat transfer.

Conversely, it is useful to consider the minimum area $A_{\min }$ as a function of the total current $I$ rather than the current density (figure 4).

It is also convenient to consider the maximum current $I_{\max }$ as a function of the total cross-sectional area of the cylinder (figure 5). These latter relations are interesting to be able to ascertain the maximum area of an arbitrary bundle, given the sum of its currents, which will be used later in this document.

The similitude between these curves and the obtained simulation curves for actual wire bundles with heterogeneous composition of materials is analyzed in [5]. Despite the heterogeneity of the wires bundles, it is possible to obtain similar curves with non-linear expressions. 


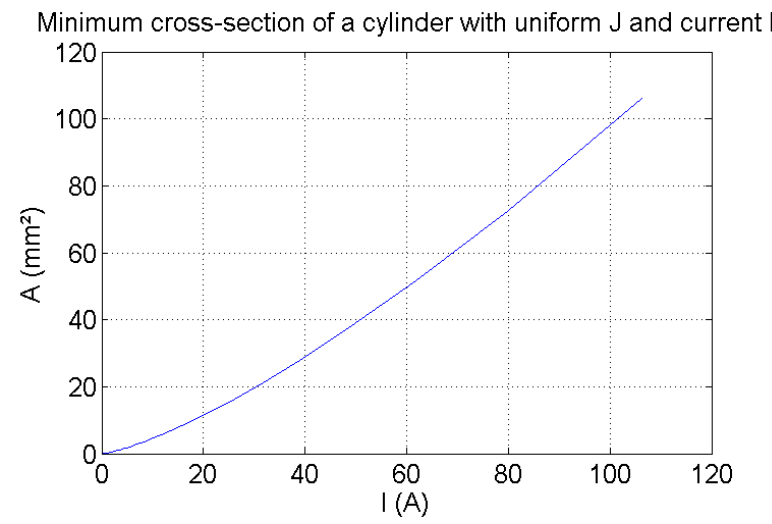

Figure 4. Minimum cross-sectional area of a cylindrical conductor subjected to a current $I$ and uniform current density. Smaller areas would produce temperatures above $T_{\max }$

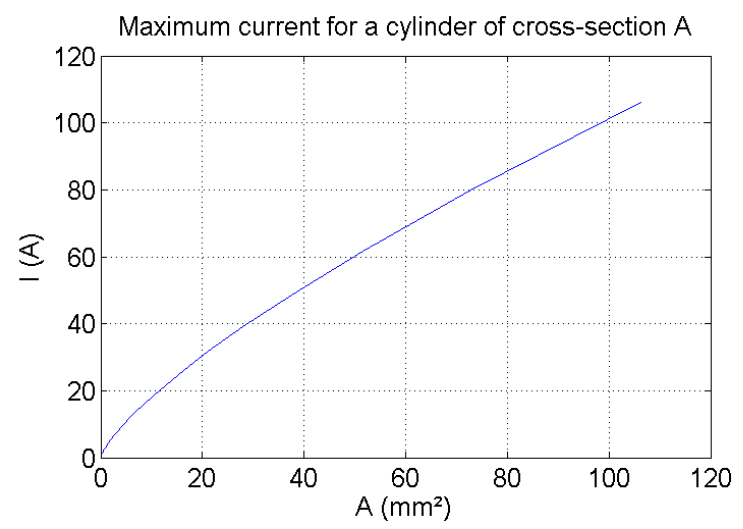

Figure 5. Maximum current for a cylindrical conductor with cross-sectional area $A$. Greater currents would produce temperatures above the maximum acceptable value

\section{REGRESSION MODELS FOR WIRE BUNDLE DIMENSIONING}

Linear regressions are carried out with the aim of obtaining general expressions for the critical area of arbitrary wire bundles. With 171 simulated cases and using the stepwise method, we obtained an expression for the logarithm of the critical area. Predictors are to the number of wires $n$, the sum of their currents, $I$ and the ambient temperature $T_{a m b}$. These predictors are included in the regression with pertinent operations such as square root or logarithm to coincide better with the theoretical formulae for cylindrical conductors discussed in [5]. This regression has obtained satisfactory results with $R^{2} \sim 1$. This can be observed in figure 6 .

\section{INTEGER OPTIMIZATION ALGORITHMS FOR CROSS-SECTION ASSIGNMENT OF WIRES IN BUNDLES}

As previously discussed, once the critical area is obtained, the cross-sections of the wires must be chosen so that the final area is minimum and greater than its critical value. If it were possible to buy wires with unconstrained cross-sections i.e. continuous and infinite availability of sizes, then the problem would be easily solved by multiplying each current by the

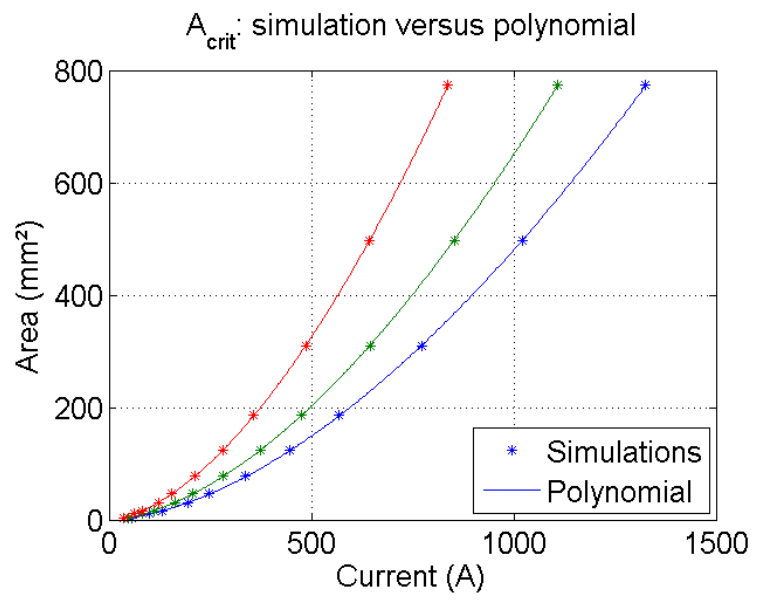

Figure 6. Regression model: minimum area of the bundle versus its sum of currents

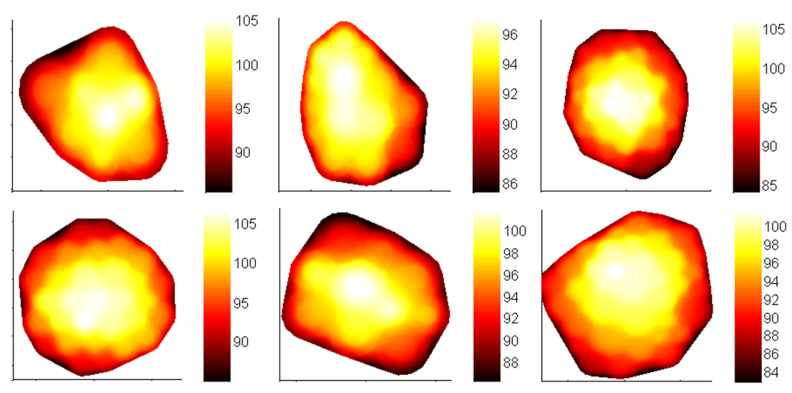

Figure 7. Simulation results of random bundles with optimized cross-sections using the linear method

obtained value of current density $J$, obtained by dividing the total current of the bundle by the sum of the areas of the wires $\left(J=\frac{I}{A}\right)$. However, cross-sections can only pertain to sets of discrete values, and it is not trivial to assign them in an optimum way. For the purpose of cross-section assignment, two different optimization algorithms have been compared. The first one is the linear binary optimization described in [5], and the second one is a custom method presented in this document.

\section{A. Integer linear programming}

The optimization algorithms assume that the critical area (obtained by means of the regression model) provides a bundle with a maximum temperature of $T_{\max }$, in this case $T_{\max }=$ $105^{\circ} \mathrm{C}$.

Wires are indexed from $k=1, \ldots, N$, being $N$ the number of wires in the bundle. In order to solve the integer optimization problem in matrix form, it is necessary to express the available cross-sections for each of the wires by means of a set of $N$ arrays called $Q_{k}$. Each of these arrays contain $S$ values of cross-sectional areas $a_{k i}(i=1,2, \ldots, S)$ for the wire $k$ (equation 4 ).

$$
Q_{k}=\left[a_{k 1}, a_{k 2}, \ldots a_{k S}\right]
$$


Each of the wires must have only one cross-section. For that reason, there are exactly $S$ boolean variables for each of the wires, i.e. there are totally $S \times N$ boolean variables (equation 5). The boolean $b_{k i}$ is true if the wire $k$ receives its cross-section $i$, and false otherwise. They are actually integer variables, and it is necessary to convert them into booleans by forcing them to be at least 0 and not higher than 1 (equation $6)$.

$$
\begin{gathered}
x=\left[b_{11}, \ldots, b_{1 S}, b_{21}, \ldots, b_{2 S}, \ldots, b_{N 1}, \ldots, b_{N S}\right]^{T} \\
x_{\min }=[0 \ldots 0]^{T} x_{\max }=[1 \ldots 1]^{T}
\end{gathered}
$$

The constraint that one wire must have only one crosssection is programmed by imposing that the sum of all of the $b_{k i}$ for the wire $k$ equals 1 :

$$
\sum_{i=1}^{S} b_{k i}=1, k=1, \ldots, N
$$

This latter constraint is expressed in matrix form as follows:

$$
\begin{gathered}
A_{e q} \cdot x=b_{e q} \\
A_{e q}=\left[\begin{array}{cccc}
1 & 1 \cdots 1 & \cdots & 0 \\
\vdots & 1 & 1 \cdots 1 & \vdots \\
0 & \cdots & 1 & 1 \cdots 1
\end{array}\right] \\
b_{e q}=[1 \ldots 1]^{T}
\end{gathered}
$$

Notwithstanding that these logical definitions are more complex, the main constraint of the problem consists of sum of all of the cross-sectional areas of the bundle not being above the critical area $\left(A_{\text {crit }}\right)$ :

$$
\sum_{k=1}^{N} \sum_{i=1}^{S} b_{k i} \cdot a_{i} \geq A_{\text {crit }}
$$

This sum of areas must be expressed in matrix form as follows, using $Q_{k}$ in eq. 4:

$$
\begin{gathered}
A \cdot x \geq A_{\text {crit }} \\
A=\left[Q_{1} \ldots Q_{k}\right]
\end{gathered}
$$

The objective function is the volume of copper, $V$ (eq. 14), since a minimized volume of copper will bring also its minimized mass, which is the real objective. This is obtained with the product of the length of each of the wires, $l_{k}$, and their cross-section, $a_{k}$, as well as their commercial mix, $w_{k}$, which is used to carry out a fleet-oriented optimization. If the optimization is only applied to one car and not to an entire fleet, these $w_{k}$ must be equal and non-zero (in that case, their recommended value would be 1).

$$
V=\left[Q_{1} \cdot l_{1} \cdot w_{1} \cdots Q_{k} \cdot l_{k} \cdot w_{k}\right] \cdot x
$$

This method is fully described in [5]. In order to validate the system, some simulations of random bundles have been performed ( [5]). Some of them can be seen in figure 7 and 8 .

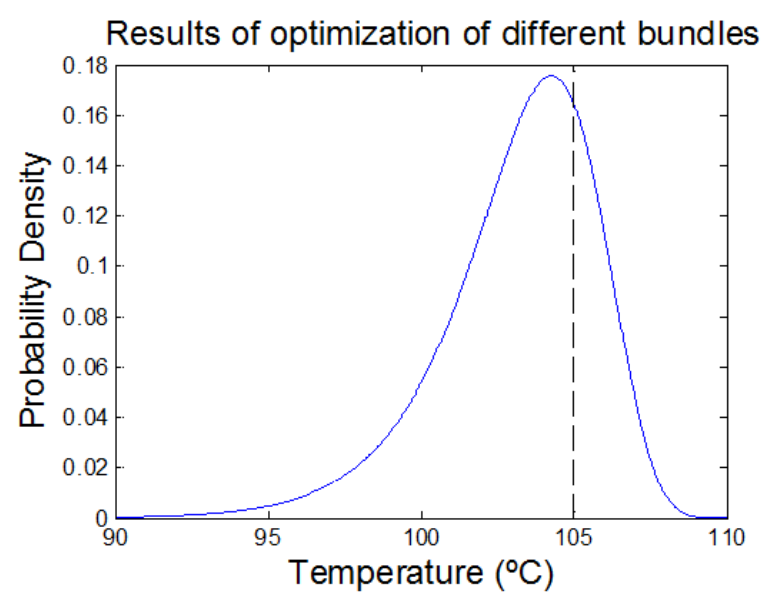

Figure 8. Fitted probability density function for maximum temperature of optimized bundles (linear method)

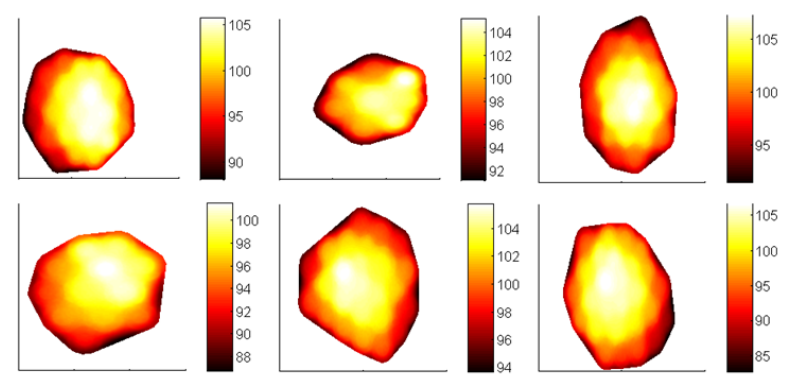

Figure 9. Simulation results of random bundles with optimized cross-sections using the custom method

\section{B. Custom method oriented to critical area}

As opposed to the optimization method described in the previous section and in [5], a custom code has been developed in order to achieve one important goal in this optimization: speed. This custom method uses the value of critical area of the bundle in order to achieve the same result as the described linear optimization, but using in this case a specific and optimization algorithm for this stated problem.

The value of critical area $A_{\text {crit }}$ is obtained from the sum of the currents of the wires, and unlike in the linear optimization method in [5], here each of the particular values of current of the wires is necessary to calculate the preliminary crosssection $a_{k}^{p r e}$, by dividing these currents by the critical current density $J_{\text {crit }}$ :

$$
\begin{gathered}
J_{\text {crit }}=\frac{\sum_{k=1}^{N} I_{k}}{A_{\text {crit }}} \\
a_{k}^{\text {pre }}=\frac{I_{k}}{J_{\text {crit }}}
\end{gathered}
$$

Using the obtained value of $J_{\text {crit }}$ each wire is given a preliminary hypothetical cross-section $a_{k}^{p r e}$, whose value is not necessarily available in the industry. Actually, from a mathematical point of view the preliminary cross-section can 
be a standard one, but this is just unlikely. For this reason, exactly as in the linear method, the gauge options of each wire are a set of industrial values of cross-section, $Q_{k}$. The algorithm could take any set of values for each of the $Q_{k}$ arrays, but its preprocessor selects the closest two standard cross-sections to $a_{k}^{\text {pre }}$ : one smaller, $a_{k}^{-}$, and one greater, $a_{k}^{+}$.

The algorithm starts assigning the lightest areas $a_{k}^{-}$to all of the wires and iteratively upgrades the cross-section of the "lightest" wire (if possible), until the sum of all of the cross-sections surpasses the critical area $A_{\text {crit }}$. The concept of lightest wire is once more related to the volume and the commercial ratio of the wires and not just to their crosssectional area. Whenever a wire is the lightest but has the thickest available cross-section, the modified wire is the second lightest, and so on and so forth.

This algorithm 1 is exposed below and uses $A_{\text {crit }}, a^{\text {pre }}, w, l$, $a^{s t}$ and $S$ as inputs. $A_{c r i t}$ is a single float value representing the critical sum of areas of the bundle. $a^{p r e}$ is an array of $N$ elements containing the preliminary cross-sectional areas of the $N$ wires. $w$ and $l$ are respectively the mixes and the lengths of each of the wires. $a^{s t}$ is the list of standard available cross-sections that these wires can take, and $S$ is the number of considered cross-sections for each wire. As previously discussed, When $S$ equals 2 , the algorithm is considering only one smaller and one greater areas for each of the $a_{k}^{p r e}$, i.e. $a_{k}^{-}$ and $a_{k}^{+}$.

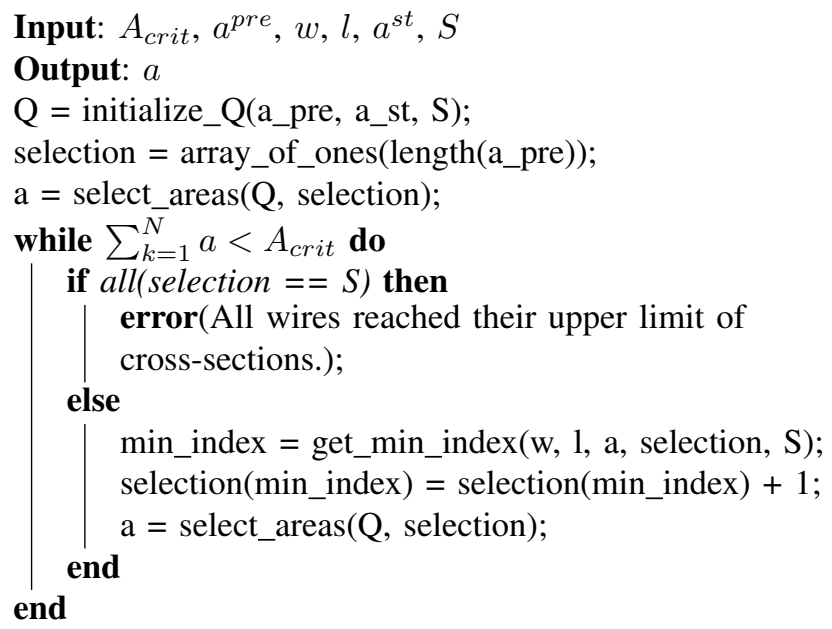

Algorithm 1: Custom optimization algorithm with critical area criterion

Twenty-four bundles with random number of wires, ambient temperature and currents have been generated. The custom optimization algorithm was used to dimension their wires, and then simulated with finite elements to observe the maximum temperature of the bundle.

The objective of the algorithm was to provide maximum temperatures lower or equal than $105^{\circ} \mathrm{C}$ with the minimum total copper volume in the bundle. Some of the simulation results can be seen in figure 9 .

The mean values of maximum temperature for the 24 simulations are $103.25^{\circ} \mathrm{C}$ for the custom method with critical

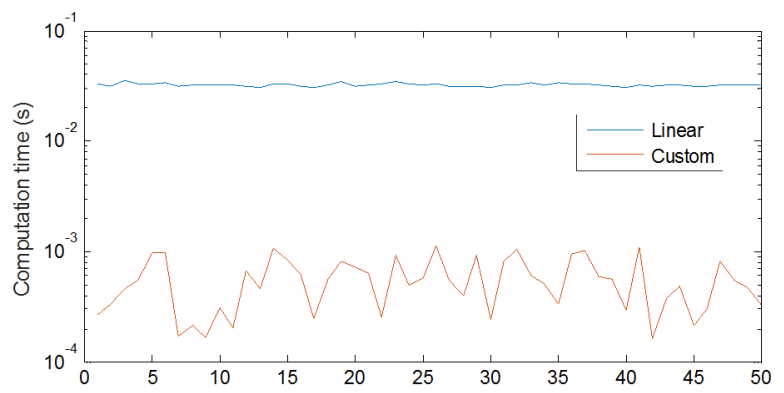

Figure 10. Comparison between computation times of the linear and the custom cross-section assignment algorithms

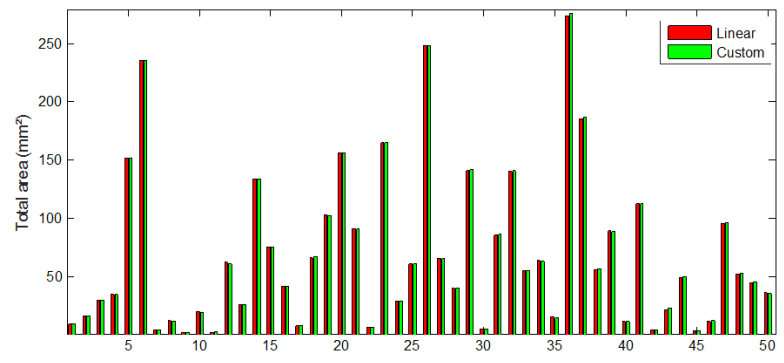

Figure 11. Comparison between obtained total conductor areas of the bundles: linear method versus custom method

area criterion. It is close to $105^{\circ} \mathrm{C}$ and it achieves the objective in average terms.

Finally, in order to compare the custom and integer linear optimization methods, fifty random wire bundle cases have been passed to these assignment algorithms. Figure 10 shows the comparison between their computation times, as well as figure 11 shows the obtained sums of cross-sectional areas. It is remarkable how the total areas are nearly identical. This suggests these two algorithms are similar in terms of copper saving.

\section{CONCLUSIONS}

In [5] expressions providing the minimum sum of crosssections of a wire bundle with given currents were obtained, as well as algorithms capable of assigning feasible crosssections to them. Results show great accordance between the models and the finite-element simulations for both the expressions of minimal area for given currents and maximum current for given area. Additionally in [5], integer linear programming is used to find the optimum dimensions of wires in bundles, taking their critical area calculated with the regression models as a constraint. Results of optimization are satisfactorily compared to finite element simulations in order to assess accordance with the constraint of critical area. In order to improve the computation time, the linear method has been replaced in this new study by a custom algorithm which also uses the critical area as a constraint. Despite the linear method provides the better results regarding maximum temperature, the custom method faster results. It is remarkable that the linear optimization method in [5] has been 
substantially improved as regards computation time, which is one main goal in this paper. However, the custom method occasionally produces temperatures above $105^{\circ} \mathrm{C}$ and presents a larger variance of temperatures among the simulated results of optimization. Therefore, it is necessary to validate these results experimentally.

\section{REFERENCES}

[1] R. Ciegis, A. Ilgevicius, H. Liess, M. Meilunas, and O. Suboc, "Numerical simulation of the heat conduction in electrical cables," Mathematical Modelling and Analysis, vol. 12, no. 4, pp. 425-439, 2007.

[2] M. Diebig and S. Frei, "Simulation-based optimization of multi voltage automotive power supply systems," in 2013 IEEE Vehicle Power and Propulsion Conference (VPPC), pp. 1-6, Oct 2013.

[3] F. Loos, K. Dvorsky, and H.-D. Liess, "Two approaches for heat transfer simulation of current carrying multicables," Mathematics and Computers in Simulation, vol. 101, pp. 13 - 30, 2014.

[4] F. Loos, H. D. Lie, and K. Dvorsky, "Simulation methods for heat transfer processes in mechanical and electrical connections," in Electric Drives Production Conference (EDPC), 2011 1st International, pp. 214-220, Sept 2011.

[5] M. A. Diaz-Millan, A. Garcia-Espinosa, and A. Rius-Rueda, "Optimization of modular wiring harnesses by means of regression models for temperature prediction of wire bundles," in Simulation and Testing for Vehicle Technology (Springer, ed.), 2016. 